\title{
Persistence of Parahippocampal Representation in the Absence of Stimulus Input Enhances Long-Term Encoding: A Functional Magnetic Resonance Imaging Study of Subsequent Memory after a Delayed Match-to-Sample Task
}

\author{
Karin Schon, ${ }^{1,2}$ Michael E. Hasselmo, ${ }^{1,2,3}$ Matthew L. LoPresti, ${ }^{2,3}$ Marisa D. Tricarico, ${ }^{2}$ and Chantal E. Stern ${ }^{1,2,3,4}$ \\ ${ }^{1}$ Department of Psychology, ${ }^{2}$ Center for Memory and Brain, and ${ }^{3}$ Program in Neuroscience, Boston University, Boston, Massachusetts 02215 , and \\ ${ }^{4}$ Athinoula A. Martinos Center for Biomedical Imaging, Massachusetts General Hospital and Harvard Medical School, Charlestown, Massachusetts 02129
}

\begin{abstract}
Recent theoretical models based on cellular processes in parahippocampal structures show that persistent neuronal spiking in the absence of stimulus input is important for encoding. The goal of this study was to examine in humans how sustained activity in the parahippocampal gyrus may underlie long-term encoding as well as active maintenance of novel information. The relationship between long-term encoding and active maintenance of novel information during brief memory delays was studied using functional magnetic resonance imaging (fMRI) in humans performing a delayed matching-to-sample (DMS) task and a post-scan subsequent recognition memory task of items encountered during DMS task performance. Multiple regression analyses revealed fMRI activity in parahippocampal structures associated with the active maintenance of trial-unique visual information during a brief memory delay. In addition to a role in active maintenance, we found that the subsequent memory for the sample stimuli as measured by the post-scan subsequent recognition memory task correlated with activity in the parahippocampal gyrus during the delay period. The results provide direct evidence that encoding mechanisms are engaged during brief memory delays when novel information is actively maintained. The relationship between active maintenance during the delay period and long-term subsequent memory is consistent with current theoretical models and experimental data that suggest that long-term encoding is enhanced by sustained parahippocampal activity.
\end{abstract}

Key words: memory; parahippocampal; neuroimaging; medial temporal lobe; delayed match to sample; computational modeling

\section{Introduction}

A number of imaging studies have demonstrated that, in humans, the medial temporal lobes (MTLs), including the parahippocampal gyri (PHG) and the hippocampus, are involved in long-term encoding of new information (Stern et al., 1996; Gabrieli et al., 1997; Brewer et al., 1998; Fernàndez et al., 1998, 1999; Kelley et al., 1998; Wagner et al., 1998; Kirchhoff et al., 2000; Otten et al., 2001). Activity in these areas has been shown to be greater during encoding of subsequently remembered than forgotten stimuli (Brewer et al., 1998; Fernàndez et al., 1998; Wagner et al., 1998; Kirchhoff et al., 2000).

Computational frameworks suggest that encoding may depend on activity being sustained in the absence of sensory input. For an item to be successfully encoded, activity at the time of

Received April 7, 2004; revised 0ct. 22, 2004; accepted 0ct. 30, 2004.

This research was supported by National Institutes of Health Grants ROI NS41636 (C.E.S.) and ROI MH61492 (M.E.H.) and a Clara Mayo Fellowship (K.S.). We thank Dr. Haline Schendan for valuable comments on and discussion of statistical analysis and preliminary results. We also thank Dr. David Somers and Murat Erdem for computational assistance. Preliminary results were presented as a poster at the 33rd Annual Meeting of the Society for Neuroscience, New Orleans, LA.

Correspondence should be addressed to Dr. Chantal E. Stern, Boston University, Center for Memory and Brain, 2 Cummington Street, Boston, MA 02215. E-mail: chantal@bu.edu.

DOI:10.1523/JNEUROSCI.3807-04.2004

Copyright $\odot 2004$ Society for Neuroscience $\quad 0270-6474 / 04 / 2411088-10 \$ 15.00 / 0$ stimulus presentation persists once the item is removed. A number of theoretical models of long-term memory (LTM) emphasize the importance of short-term persistence of representations for encoding (Atkinson and Shiffrin, 1968; Jensen and Lisman, 1996; Hasselmo et al., 2002b; Howard and Kahana, 2002). Recent models have used persistent spiking in parahippocampal structures to hold input to allow encoding of associations between behavioral stimuli separated in time by long delays (Jensen and Lisman, 1996; Hasselmo et al., 2002a,b; Koene et al., 2003). These models are based on evidence that single neurons in parahippocampal structures have intrinsic currents activated by muscarinic cholinergic receptors that allow them to maintain sustained spiking activity that does not depend on previous modification of synaptic connections (Klink and Alonso, 1997; Fransén et al., 2002). This sustained activity allows the models to use spiketiming-dependent synaptic plasticity with short time delays (Levy and Steward, 1983; Markram et al., 1997; Bi and Poo, 1998) to associate stimuli separated across time.

The persistent activity associated with a stimulus can be analyzed in delayed match-to-sample tasks, in which a subject encounters a sample stimulus that is followed by a delay period before presentation of a test stimulus. The subject is required to make a response that indicates that the subject recognized this test item as either matching [delayed match-to-sample (DMS)] 
or not matching [delayed-nonmatch-to-sample (DNMS)] the preceding sample. Sustained neural activity has been observed in cortical structures, including entorhinal cortex (ERC), during the delay period when there is no perceptual stimulation (Suzuki et al., 1997; Young et al., 1997). This delay activity could contribute to long-term encoding, particularly if it appears for novel stimuli. Recently, neuroimaging studies have reported significant MTL activity during performance of delayed matching tasks with trialunique stimuli but not with familiar stimuli (Ranganath and D'Esposito, 2001; Stern et al., 2001). We set out to test in humans a hypothesis based on the computational models, namely that sustained activity in the MTL, and in particular within the parahippocampal gyrus, would enhance the successful encoding of novel information into LTM in subjects performing a DMS task. We addressed this question by combining measurement of activity during the delay period of a DMS task during functional magnetic resonance imaging (fMRI) with a post-scan subsequent recognition memory task.

\section{Materials and Methods \\ Participants}

Sixteen healthy individuals (six male; mean age, $23 \pm 5$ years) were recruited from the student community at Boston University. No subjects reported any neurological or psychiatric history. All subjects were screened for MRI compatibility. Vision was normal or corrected-tonormal. All subjects gave signed informed consent before participating in this study in accordance with the Human Research Committee of the Massachusetts General Hospital and the Institutional Review Board of Boston University.

\section{Procedure}

We used trial-unique complex visual scenes [stimuli depicting indoor scenes, $n=191$; stimuli depicting outdoor scenes, $n=172$ (Stern et al., 1996, 2001; Kirchhoff et al., 2000; Sherman et al., 2003)] as stimuli. Sample and test stimuli were shown for 2 sec each, with a memory delay of $10 \mathrm{sec}$ between sample and test presentation (Fig. 1A). Subjects also performed a visuomotor control task $(\mathrm{CON})$ that did not require any memory retention during the delay period and that differed from the DMS task only in instruction (Fig. $1 \mathrm{~A}$ ). The CON task simply required the subjects to judge whether the test picture was indoors or outdoors. Approximately $20 \mathrm{~min}$ after fMRI scanning, subjects performed a surprise subsequent recognition memory test (SMT) in which they were asked to rate on a five-point scale (Sherman et al., 2003) their confidence level that studied pictures and unstudied lures were old or new.

DMS and CON tasks. We used a DMS task with trial-unique complex visual scenes as stimuli (digitized photographs; $10.23 \times 6.81 \mathrm{~cm}$ ) to assess active maintenance and encoding during short memory delays. Additionally, we used a CON task that differed from the DMS task only in instruction. Stimuli for this task were randomly selected from the same stimulus pool as those used in the DMS task. In both tasks, each trial consisted of a $2 \mathrm{sec}$ visual scene presentation (sample), followed by a 10 sec delay period, followed by a $2 \mathrm{sec}$ visual scene presentation (test), followed by a variable-length intertrial interval (ITI) $(6,10$, or $14 \mathrm{sec})$ (Fig. $1 \mathrm{~A}$ ). The delay period was similar in length to previous fMRI working memory studies using delayed matching procedures (Courtney et al., 1998; Postle and D’Esposito, 1999a). The variable ITI introduces temporal "jitter" for selective averaging and provides time for the hemodynamic response to return to baseline (Postle et al., 2000). The length of the task delays was kept fixed because different memory-delay lengths have been shown previously to result in qualitatively different activation patterns (Elliott and Dolan, 1999). During the delay, subjects viewed a mask composed of a gray box of the same dimensions as the visual scenes and a black fixation cross in the center. A cue was presented during sample, delay period, and test presentation to instruct the subject. The cue "MATCH" instructed the subject to remember the sample picture during the delay period. During the test, the cue changed to "MATCH?", prompting the subject to make a yes-no response on whether or not the test picture matched the sample using a button box (DMS task). Half of

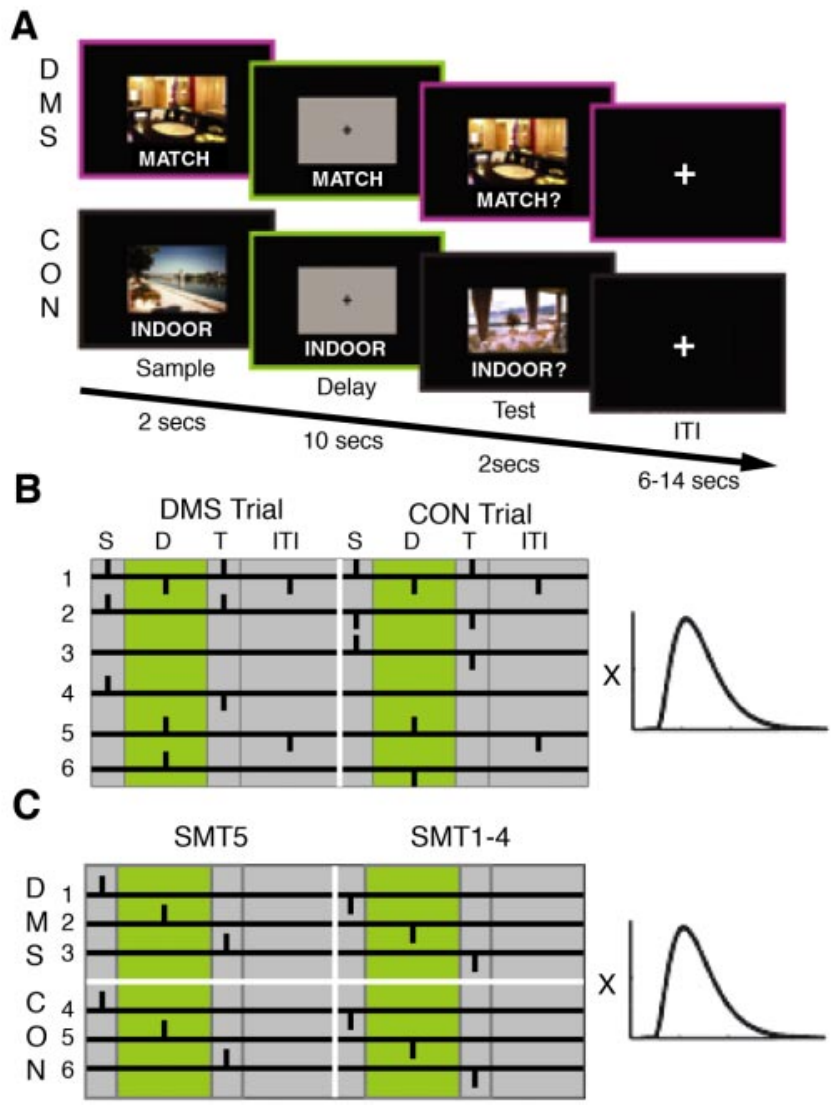

Figure 1. Tasks and statistical analyses of $\mathrm{fMRI}$ data. A, DMS and CON. In both tasks, a trial consisted of three time-locked events: a 2 sec visual scene presentation (sample), followed by a 10 sec delay period, followed by a 2 sec visual scene presentation (test), followed by a variablelength intertrial interval. Tasks differed only in instruction. $B$, Contrasts for active maintenance analysis. C, Contrasts for subsequent memory analysis. Regressors were created by convolving the six orthogonal contrasts of interest (depicted on the left) with a gamma-variate function (HRF; depicted on the right) (Boynton et al., 1996). Vertical bars represent positioning of the HRF. S, Sample; D, delay; T, test. For details, see Materials and Methods.

the trials were matches (“yes" responses), and half were nonmatches ("no" responses). The order of match and nonmatch trials was counterbalanced. The cue "INDOOR" prompted the subject to simply wait until the test picture appeared. During the test, the cue changed to "INDOOR?", prompting the subject to indicate whether or not the picture was indoors (CON task). Half of the test pictures depicted indoor scenes ("yes" responses), and half depicted outdoor scenes ("no" responses). No reference back to the sample picture was necessary to perform this task accurately. The CON task sample picture did not predict whether the test picture was indoors or outdoors. The order of indoor and outdoor trials was counterbalanced. In these tasks, memory was maximized during the delay period of the DMS task and minimized during the delay period of the CON task, while keeping visuomotor requirements constant. During scanning, subjects performed eight functional runs, consisting of six DMS and six CON trials each. The order of DMS trials and CON trials, and trials requiring "yes" and "no" responses, was counterbalanced within runs. Approximately 50\% of the pictures seen during DMS and CON task performance depicted indoor scenes. We designed 12 parallel versions of a set of eight runs, each of which included all counterbalancing and randomization requirements to ensure that no more than two subjects would perform the exact same set of tasks. Before scanning, subjects viewed task instructions on a computer and practiced the tasks.

Subjects were instructed to respond as quickly and as accurately as possible. Stimuli were presented, and reaction times (RTs) and errors were recorded with PsyScope 1.2.5. (Cohen et al., 1993).

Post-scan SMT. Approximately 20 min after fMRI scanning, subjects performed a surprise self-paced SMT. Subjects viewed all stimuli pre- 
sented as samples and tests during both DMS and CON trials $(n=118)$ and an equal number of new pictures in random order. They were instructed to rate on a five-point scale their confidence about whether or not a displayed scene was previously presented during the DMS and CON tasks. The rating scale included "high-confidence new" (1), "lowconfidence new" (2), "unsure" (3), "low-confidence old" (4), and "highconfidence old" (5).

\section{fMRI data acquisition}

Data were acquired on a 3.0 Tesla Allegra Siemens (Erlangen, Germany) scanner at the Martinos Center at the Massachusetts General Hospital. Two high-resolution T1-weighted structural images were acquired for each subject (MP-RAGE; field of view, $256 \times 256 ; 128$ slices). We ran eight functional $\mathrm{T} 2{ }^{\star}$-weighted gradient-echo, echo-planar blood oxygenation level-dependent (BOLD) scans [repetition time (TR), 2 sec; echo time, $30 \mathrm{msec}$; flip angle, $90^{\circ}$; field of view, $64 \times 64$; 21 slices] and acquired 149 images during each scan per slice $(3.1 \times 3.1 \mathrm{~mm} ; 5 \mathrm{~mm}$ slice thickness; $1 \mathrm{~mm}$ skip between slices). Slices were aligned parallel to the anterior commissure-posterior commissure line to acquire data from the whole brain.

\section{fMRI data preprocessing}

Using SPM2 (Wellcome Department of Cognitive Neurology, London, $\mathrm{UK})$, functional images were corrected for differences in slice timing, realigned to the first image within a series, unwarped to correct for susceptibility-by-movement interactions, normalized into MNI (Montreal Neurological Institute) space, which included SPM standard resampling to $2 \times 2 \times 2 \mathrm{~mm}$ isotrophic voxels, and spatially smoothed using a $6 \mathrm{~mm}$ Gaussian filter.

\section{fMRI statistical analysis}

Active maintenance analysis. Active maintenance during memory delays was assessed using single-subject multiple regression analysis with orthogonal regressors. This methodological approach has been described previously (Zarahn et al., 1997; Postle et al., 2000) and used (Courtney et al., 1997, 1998; Postle and D'Esposito, 1999a,b) to assess delay period activity in data generated from subjects performing a delayed matching task while in the fMRI scanner. With multiple regression, hemodynamic changes to different trial components that are segregated in time, but that follow in a specific order (e.g., sample, followed by a delay, followed by a test), can be analyzed simultaneously and independently (Courtney et al., 1998). This is important for the analysis of DMS tasks, because it is impossible to randomize and counterbalance the placement of the individual events because of their time-locked nature, which is an important necessity for conventional rapid "event-related" (i.e., trial-based) fMRI analysis (Dale and Buckner, 1997; Buckner, 1998). Similar to Courtney et al. (1998), we created six orthogonal contrasts that reflected comparisons of interest and convolved them with a gamma variate function [hemodynamic response function (HRF)] (Boynton et al., 1996). We created the following six regressors (Fig. $1 B$ ): regressor 1 assessed activation resulting from visual stimulation versus no visual stimulation; regressor 2 assessed the difference in activation resulting from the type of stimuli (DMS stimuli vs CON stimuli); regressor 3 assessed the difference between sample stimulus presentation versus test stimulus presentation and motor response for $\mathrm{CON}$ stimuli; regressor 4 assessed the difference between sample stimulus presentation versus test stimulus presentation and motor response for DMS stimuli; regressor 5 assessed differences in activity during task delays (across task) versus during the ITI; and regressor 6 assessed the difference in activity during the DMS task delay and the CON task delay. Following the methods of Postle et al. (2000), we used "stick" contrasts to position the HRF to mitigate collinearity not only among the unconvolved contrasts but also among the convolved regressors. This method has been used previously for fMRI data analysis for the assessment of delay period activity (Postle and D'Esposito, 1999a,b; Postle et al., 2000) and is important because, if two regressors (contrasts that have been convolved with the HRF) or contrasts (unconvolved) share a significant amount of variance, then the results for one regressor cannot be interpreted independently of the other (Cohen and Cohen, 1983; Bortz, 1993). Each pair of regressors or contrasts is orthogonal if the sum of the products of their weight coefficients is zero (Cohen and
Cohen, 1983; Bortz, 1993). Vertical bars in Figure $1 B$ represent the positioning of the HRFs before convolution, and all six (convolved) regressors in this study were orthogonal or nearly orthogonal. This approach allowed us to isolate activity that was solely attributable to the delay component of the task (regressor 6) (Postle et al., 2000). All six regressors were entered simultaneously into SPM2 as covariates. Statistical analysis in SPM2 included overall grand mean scaling to 100, proportional threshold masking, and global calculation of mean voxel value within per image full mean/eight mask. One SPM $\{\mathrm{T}\}$ map was generated for each regressor for each subject.

Subsequent memory analysis. Long-term encoding was assessed by selectively averaging sample presentation periods and test presentation periods according to whether or not a subject remembered a given sample or test, respectively, with high confidence during the post-scan subsequent memory test (SMT5 vs SMT1-4). Delay period activity was assessed by selectively averaging delay periods according to whether or not the preceding sample was remembered with high confidence (SMT5 vs SMT1-4). To adapt selective averaging for multiple regression analysis, six orthogonal contrasts were created (Fig. 1C) and convolved with the HRF (Boynton et al., 1996). As for the active maintenance analysis, the six convolved contrasts constituted the six regressors for this analysis. Three regressors were created for each of the two tasks. Regressors corresponded to the three components of each trial: the stimulus sample period (SAMPLE), the delay period (DELAY), and the match versus non-match test period (TEST). The first regressor contrasted sample presentation periods for items that were subsequently remembered with high confidence (SMT5) versus all other ratings (SMT1-4). The second regressor contrasted delay periods after pictures that were remembered with high confidence (SMT5) versus all other responses (SMT1-4). The third regressor contrasted test presentation periods of test pictures that were subsequently remembered with high confidence (SMT5) versus all others (SMT1-4). We contrasted SMT5-related activity with SMT1-4related activity, because we were interested in recollection-based, not in familiarity-based, behavioral responses on the SMT to study long-term episodic encoding. A response of 5 on the SMT is more likely to be recollection based than a response of 4 (Yonelinas, 1994; Sherman et al., 2003). We chose the contrasts such that encoding-related activity attributable to sample presentation could be assessed separately during sample and delay periods and independently from other trial components. The convolved regressors were orthogonal or nearly orthogonal to ensure interpretability of results.

An additional subsequent memory multiple regression analysis was performed to exclude "match" trials and only assess subsequent memory effects separately for DMS "nonmatch" trials. This analysis was done because match trials involve two presentations of the same sample stimulus, whereas nonmatch trials involve just one single presentation. In our study, although delay activity during match and nonmatch trials should not differ if the subject has no information on the outcome of the trial during the delay while performing the task, this activity can differ if the delays are sorted later according to responses on the SMT, because activity during the delay can influence subsequent memory. Behavioral performance on the SMT further indicated a quantitative difference between the proportion of " 5 " responses to DMS sample stimuli that were matches (presented twice) and the proportion of " 5 " responses to DMS sample stimuli that were nonmatches (presented once; Fig. 2, compare $E$, $F$ ). Subsequent memory analysis with nonmatch trials was performed to avoid this confound of number of stimulus presentations. This is the most crucial analysis of our study for examining delay related activity associated with subsequent memory after the 20 min delay in the SMT task. Regressors were again created to examine sample, delay, and test periods, but, in this case, stimuli were divided into match (two presentations) and nonmatch (single presentation) trials. The first regressor contrasted sample presentation periods of sample pictures for DMS match trials that were subsequently remembered with high confidence (SMT5) with those that were not (SMT1-4). The second regressor examined the same sample periods for nonmatch trials, the third contrasted delay periods that immediately followed sample pictures for match trials that were subsequently remembered with high confidence (SMT5) versus delay periods after all other sample pictures (SMT1-4), 


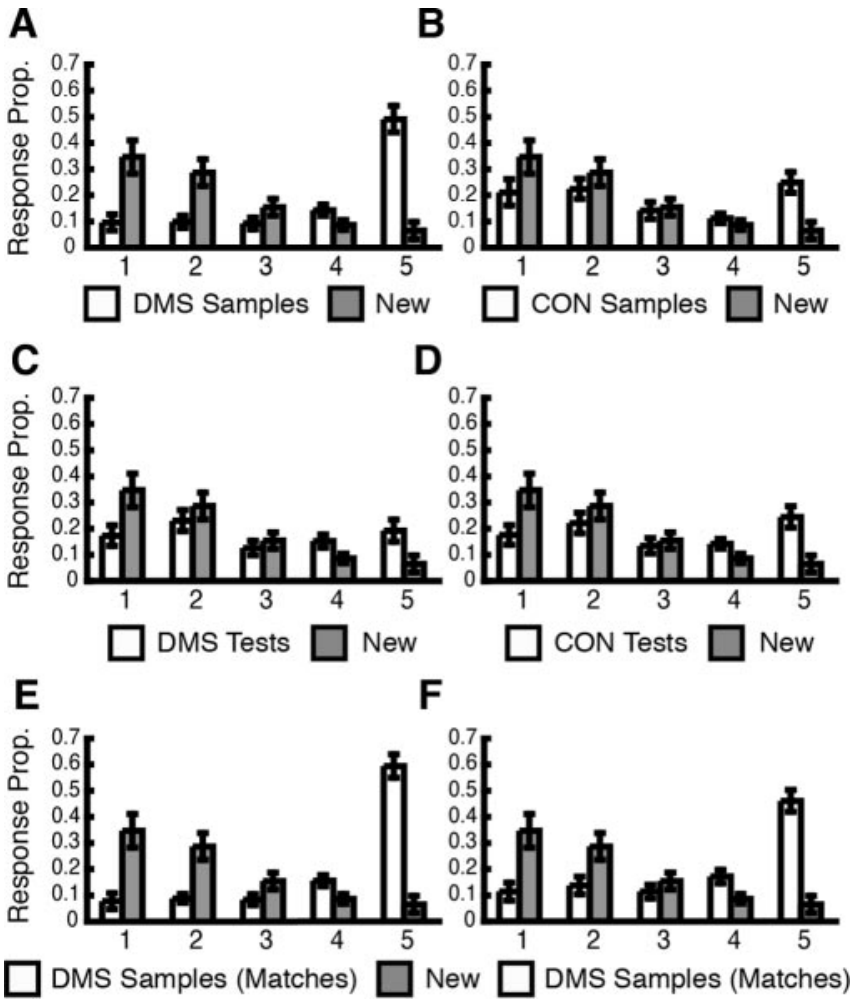

Figure 2. Behavioral results of subsequent memory rating. Mean \pm SE proportion responses is depicted on $y$-axes, and confidence rating (5, high-confidence old; 4 , low-confidence old; 3 , unsure; 2 , low-confidence new; 1 , high-confidence new) is depicted on $x$-axes. A, DMS samples versus new stimuli (lures; gray bars). B, CON samples versus new stimuli. C, DMS tests versus new stimuli. $D$, CON tests versus new stimuli. $E$, DMS samples (match trials only) versus new stimuli. F, DMS samples (nonmatch trials only) versus new stimuli.

and the fourth examined the same for nonmatch trials. All other regressors did not change (Fig. 1C, regressors 3-6). This subsequent memory analysis limited to nonmatch trials, which only included stimuli that were presented once, allowed us to compare our data with those from previous long-term encoding studies in which stimuli were presented only once (Stern et al., 1996; Brewer et al., 1998; Kirchhoff et al., 2000).

Random-effects group analyses with group as the random factor were performed with SPM2 on each regressor by entering the t-contrast images of each subject corresponding to a particular regressor into a second-level one-sample $t$ test.

Region-of-interest analyses. We defined our temporal lobe region of interest (ROI) structurally as composed of the parahippocampal gyri [including parahippocampal, perirhinal (PRC), and entorhinal cortices], the hippocampi, and the fusiform gyri (FG), bilaterally, because these regions have been reported to show subsequent memory effects in studies of long-term encoding. An ROI mask was created using an atlas-based tool, WFU_PickAtlas (Maldjian et al., 2003), in conjunction with SPM2 to perform small-volume corrections using a corrected statistical threshold of $p<0.05_{\mathrm{COR}}$, with a threshold extent of five voxels. The WFU_PickAtlas tool allows the generation of atlas-based ROI masks using multiple different atlases, including the ICBM (International Consortium for Brain Mapping) atlas (Mazziotta et al., 2001) that was used to define the subregions of the medial temporal lobes and create the ROI mask used here.

Time-series analyses. Signal intensity time series were extracted from activation peaks of both active maintenance and subsequent memory analyses from each subject. We used the volume of interest (VOI) tool in SPM2 to extract the signal intensity data from voxels that fell within a 5 $\mathrm{mm}$ sphere with the ROIs peak activation at its center. Signal intensities (Y) were adjusted for effects of interest and were extracted for each time point (149 time points per BOLD scan) and selectively averaged by task (DMS vs CON), by post-scan subsequent memory (SMT5 vs SMT1-4), and by event (sample vs delay vs test). Time series were statistically analyzed using repeated-measures ANOVAs with the within-subject factors task (DMS vs CON), subsequent memory (SMT5 vs SMT1-4), and event (sample vs delay vs test periods). For the subsequent memory analysis of nonmatch trials, time series of DMS trials were statistically analyzed using repeated-measures ANOVAs with the within-subject factors DMS trial (match vs nonmatch), subsequent memory (SMT5 vs SMT1-4), and event (sample vs delay vs test periods). The event factor included three time points: one time point corresponding to the TR of the sample period, one time point corresponding to the mean of the five TRs of the delay period, and one time point corresponding to the TR of the test period. Geisser-Greenhouse (Geisser and Greenhouse, 1958) degrees-offreedom corrections that correct for nonhomogenous correlations and variances among factor levels were performed, and corresponding $p$ values were reported, if applicable.

Behavioral data analysis. $t$ tests compared DMS and CON tasks on two dependent variables: average median RTs and average accuracy.

A repeated-measures ANOVA and two-tailed $t$ tests (Bonferroni corrected) with the within-subject factors event (DMS sample matches, DMS sample nonmatches, DMS test nonmatches, CON samples, CON tests, and new stimuli) and confidence rating (1, high-confidence new; 2 , low confidence new; 3 , unsure; 4 , low confidence old; and 5, high confidence old) assessed task performance on the SMT. Geisser-Greenhouse (Geisser and Greenhouse, 1958) degrees-of-freedom corrections were performed, and corresponding $p$ values were reported, if applicable.

All behavioral analyses used a statistical threshold of $p<0.05$ (Bonferroni corrected).

\section{Results}

\section{Behavioral performance}

DMS and CON performance

The average \pm SD accuracy on DMS trials was $0.996 \pm 0.009$ and on CON trials was $0.969 \pm 0.035$. RT and accuracy data were not included for one subject because of technical problems. On average, subjects made less than one error per run in both DMS and CON trials; however, a one-sample $t$ test revealed a statistically significant effect of task $\left(t_{(14)}=2.966 ; p=0.010\right)$, showing that, on average, more errors were made during CON trials than during DMS trials, but the effect was small (Cohen's $d=1.033$ ), indicating a difference of one pooled SD. Similarly, subjects performed slightly, but significantly, faster in DMS trials $\left(M_{\mathrm{WM}}=\right.$ $908.414 \pm 263.015)$ than in CON trials $\left(M_{\mathrm{CON}}=1121.952 \pm\right.$ $\left.272.680 ; t_{(14)}=-9.814 ; p<0.001\right)$. Again, this effect was small (Cohen's $d=0.798$ ), indicating a difference of less than one pooled SD. Overall, CON trials were slightly more difficult than DMS trials. This significant difference between the two tasks was likely attributable to the ceiling performance on the DMS task that resulted in reduced variance.

\section{Post-scan subsequent memory rating}

Distributions of average response proportions (mean $\pm \mathrm{SE}$ ) are depicted in Figure 2. A repeated-measures ANOVA revealed a significant main effect of confidence rating $\left(F_{(4,60)}=4.83 ; p<\right.$ $0.01)$ and a significant event by confidence rating interaction $\left(F_{(20,300)}=20.01 ; p<0.001\right)$. Tests of within-subject simple contrasts revealed significant 2 by 2 interactions between all but one event and memory combinations. Statistical significance levels obtained from the ANOVA did not change when nonsphericity was corrected using the Geisser-Greenhouse procedure. Paired-sample $t$ tests comparing confidence ratings of five responses (high confidence old) for pairs of events revealed that the response proportions for DMS sample nonmatches, DMS sample matches, DMS test nonmatches, CON samples, and CON tests were significantly higher than that for new stimuli $\left(t_{(15)}=7.72\right.$; $t_{(15)}=6.62 ; t_{(15)}=3.67 ; t_{(15)}=4.49$; and $t_{(15)}=5.11$, respec- 
tively; $p<0.003$ ), indicating successful memory encoding during both DMS and CON trials.

As expected, mean response proportions for SMT5 (high confidence old) ratings were highest for DMS sample matches $(0.59 \pm 0.04)$ and DMS sample nonmatches $(0.46 \pm 0.04)$, followed by CON samples $(0.25 \pm 0.04)$, CON tests $(0.25 \pm 0.04)$, and DMS test nonmatches $(0.21 \pm 0.04)$, and were lowest for new stimuli $(0.06 \pm 0.03)$. The response proportions (Fig. 2A,E,F) for DMS samples are comparable with recently reported results from a behavioral long-term encoding study that used the same SMT paradigm with complex visual scenes (Sherman et al., 2003).

\section{fMRI results}

Active maintenance and subsequent memory are closely related

We investigated active maintenance by contrasting DMS and CON delays regardless of whether subjects subsequently remembered the pictures (Fig. $1 B$, regressor $6)$. This analysis revealed that, when DMS delays were contrasted with $\mathrm{CON}$ delays (Fig. $1 B$, regressor 6 ), the mid-FG/PHG was activated on the right (peak at $x=34$, $\left.y=-34, z=-20 ; Z=4.18 ; p<0.05_{\mathrm{COR}}\right)$ (Fig. 3A) and on the left (peak at $x=-32$, $\left.y=-48, z=-12 ; Z=3.60 ; p<0.05_{\mathrm{COR}}\right)$ (Fig. $3 D$ ). The activation peaks are within the mid-FG on both sides and extend medially into the banks of the collateral sulcus, which is part of the posterior PHG (Pruessner et al., 2002). Because of variability of the collateral sulcus in individual subjects, additional analyses at the singlesubject level were performed and demonstrated that both the fusiform and parahippocampal gyri were active in most subjects. Supplemental data providing information about parahippocampal activation in individual subjects for this comparison is available at www.jneurosci.org as supplemental material.

Repeated-measures ANOVAs on the VOI time series in these ROIs in right and left mid-FG/PHG revealed a significant main effect of event (sample vs delay vs test periods) $\left[F_{(2,165)}=6.28, p=0.013\right.$ (Fig. $3 B$ ); and $F_{(2,165)}=41.6, p<0.0001$ (Fig. $3 E$ ), respectively] and a subsequent memory effect in the right mid-FG/PHG ROI for DMS trials, as indicated by a trend for a task (DMS $\geq \mathrm{CON}$ ) by memory $\left(\right.$ SMT5 $>$ SMT1-4) interaction $\left[F_{(1,165)}=2.91 ; p=\right.$ 0.090 (Fig. 3C) ]. A repeated-measures ANOVA on the VOI time series in the left mid-FG/PHG revealed a significant main effect of task [DMS $>$ CON; $F_{(1,165)}=7.97 ; p=0.005$ (Fig. 3E)], a significant main effect of memory [SMT5 $<$ SMT1-4 for CON tests; $F_{(1,165)}=4.47 ; p=0.036$ (data not depicted)], and a trend toward a task (DMS vs CON) by event (sample vs delay vs test periods) interaction $\left[F_{(2,165)}=2.97 ; p=0.080\right.$ (Fig. $3 E$ ) $]$. To summarize,
A RIGHT MID-FG/PHG

B RIGHT MID-FG/PHG

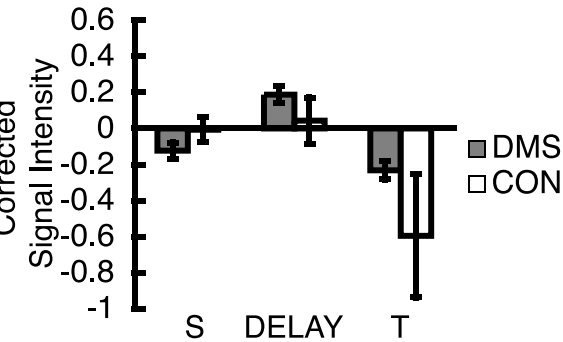

C RIGHT MID-FG/PHG

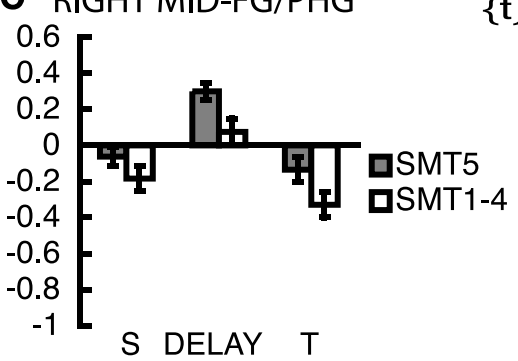

$\{t\}$

\section{LEFT MID-FG/PHG}
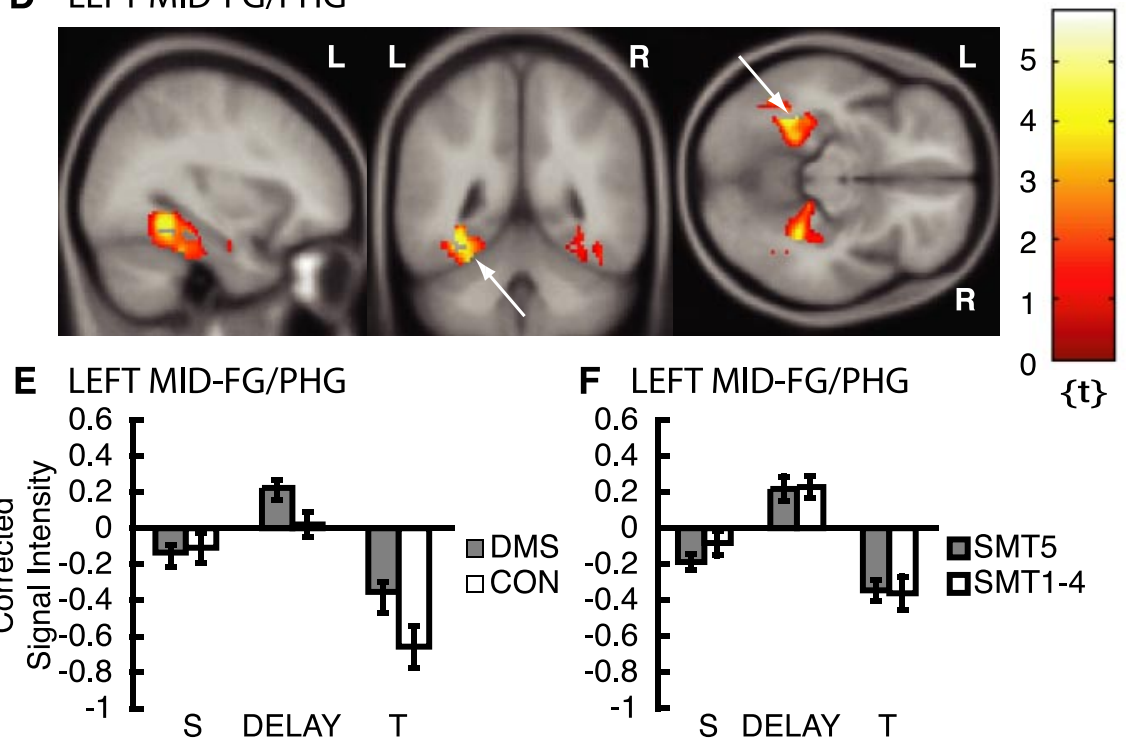

Figure 3. $\quad f M R I$ results from active maintenance analysis. Please note that only activation within ROls is superimposed on canonical average T1-weighted ICBM/MNI brain. A, Right mid-FG/PHG, $x=34, y=-34, z=-20$ (arrows). $B$, Corrected signal intensities during sample presentation (S), delay period (DELAY), and test presentation (T) from right mid-FG/PHG; DMS $>$ CON. C, Corrected signal intensities from right mid-FG/PHG; SMT5 $>$ SMT1-4. D, Left mid-FG/PHG, $x=-32, y=-48, z=-12$ (arrows). E, Corrected signal intensities from left mid-FG/PHG; DMS $>$ CON. F, Corrected signal intensities from left mid-FG/PHG; SMT5 > SMT1-4. y-Axes, signal intensity grand mean scaled to 100 and global calculation using mean voxel value (within per image full mean/eight mask). R, Right; L, left.

activity during the memory delay in the right mid-FG/PHG was greater for samples that were subsequently remembered with high confidence than for samples that were not (Fig. 3C). Activity was greater during DMS task delays than during CON task delays in the left mid-FG regions regardless of post-scan subsequent memory (Fig. $3 E, F$ ).

Additional foci of activation in the comparison of DMS delays versus CON delays included the right inferior frontal gyrus (BA45/46), the right and left precentral gyri (BA4/6), the right and left uncal apex (BA28) medial to hippocampal head and amygdala, the right posterior inferior temporal gyrus (BA37), the right and left superior parietal gyri (BA7), the right intraparietal sulcus, and the left inferior occipital gyrus (BA37) $(p<0.01$, uncorrected; threshold extent, 5 voxels). 
A RIGHT PHG/MID-FG

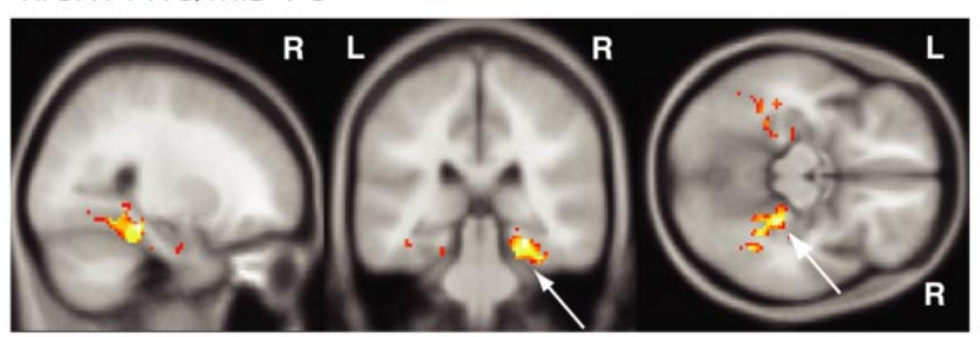

B RIGHT PHG/MID-FG

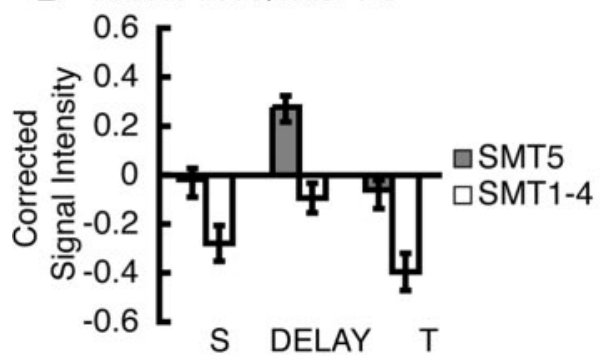

C RIGHTPHG/MID-FG

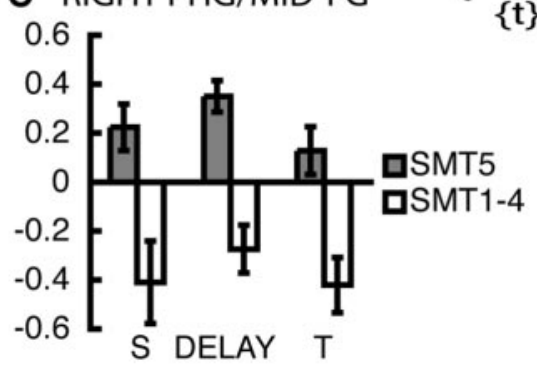

D RIGHT PRC/ERC

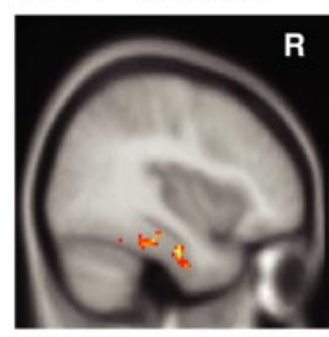

R L

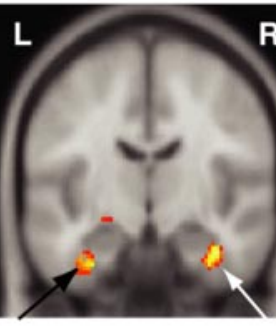

$\mathbf{R}$
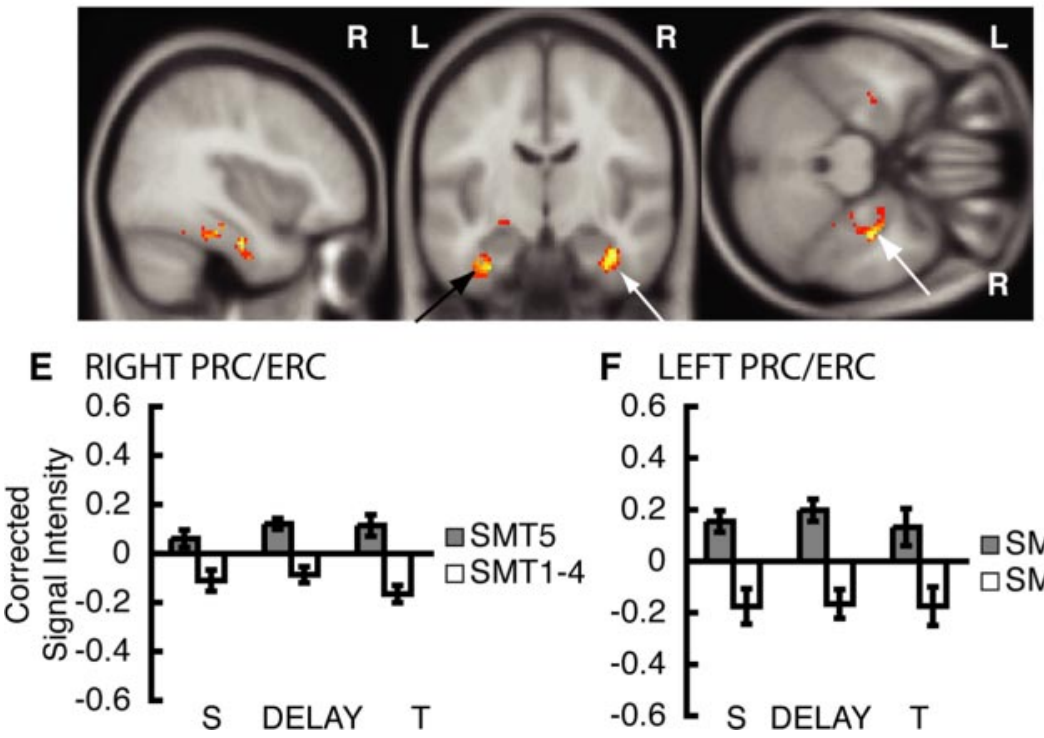

0.6

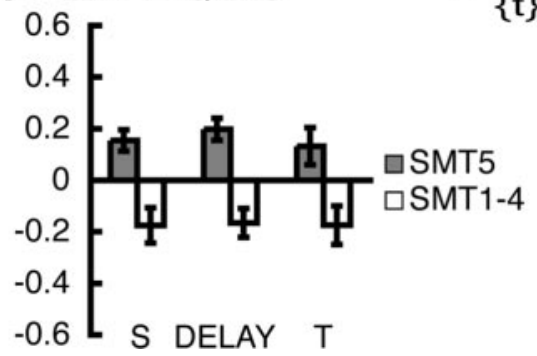

Figure 4. fMRl results from subsequent memory analysis across match and nonmatch trials (double or single stimulus presentations). Please note that only activation within ROIs is superimposed on canonical average T1-weighted ICBM/MNI brain. A, Right PHG/mid-FG, $x=26, y=-32, z=-14$ (arrows). $B$, Corrected signal intensities during sample presentation (S), delay period (DELAY), and test presentation (T) from right PHG/mid-FG for DMS trials (across match and nonmatch trials, double or single stimulus presentations); SMT5 > SMT1-4. C, Corrected signal intensities from right PHG/mid-FG for DMS trials that were nonmatches (single stimulus presentations); SMT5 $>$ SMT1-4.D, Right PRC/ERC, $x=38, y=-12, z=-26$ (white arrows); and left PRC/ERC, $x=-28, y=-8, z=-34$ (black arrow). E, Corrected signal intensities from right PRC/ERC for DMS trials (across match and nonmatch trials, double or single stimulus presentations). F, Corrected signal intensities from left PRC/ERC for DMS trials (across match and nonmatch trials). $y$-Axes, Signal intensity grand mean scaled to 100 and global calculation using mean voxel value (within per image full mean/eight mask). $R$, Right; $L$, left.

Delay activity predicts long-term subsequent memory

The subsequent memory multiple regression analysis contrasted selectively averaged DMS delays with preceding samples that were subsequently remembered with high confidence (SMT5) versus selectively averaged DMS delays with preceding samples that were not (SMT1-4) (Fig. 1C, regressor 2) to further explore the subsequent memory effect in the right mid-FG/PHG. Figure $4 A$ shows that an area within the right posterior PHG that extended into the mid-FG (PHG/mid-FG) predicted subsequent memory (peak at $x=26, y=-32, z=-14 ; Z=3.47 ; p<$
$0.05_{\mathrm{COR}}$. $)$ This area is similar in location to the one from the active maintenance analysis, but the peak is more medial and lies within the posterior PHG (Pruessner et al., 2002). Additional analyses at the single-subject level revealed parahippocampal activity in the majority of subjects. Information about parahippocampal activation in individual subjects for this comparison is provided in a supplemental data section available at www. jneurosci.org as supplemental material. A VOI time-series repeated-measures ANOVA in the right PHG/mid-FG ROI revealed a subsequent memory effect in the DMS delay as indicated by a significant main effect of memory $\left[F_{(1,165)}=9.95\right.$; $p=0.002$ (Fig. $4 B$ ) ] and by a significant main effect of event [sample vs delay vs test; $F_{(2,165)}=7.60 ; p=0.001$ (Fig. $4 B$ )]. Figure $4 D$ shows additional activation peaks within the banks of the anterior collateral sulcus, in the perirhinal cortex, and in the lateral aspect of the entorhinal cortex (right peak at $x=-38, y=-12, z=$ $-26, Z=3.20, p<0.05_{\mathrm{COR}}$ (Fig. $4 D$, white arrows); left peak at $x=-28, y=$ $-8, z=-34, Z=2.91, p<0.05_{\mathrm{COR}}$ (Fig. $4 D$, PRC/ERC, black arrow) (Insausti et al., 1998; Pruessner et al., 2002). A VOI time-series repeated-measures ANOVA in these regions showed a long-term subsequent memory effect as indicated by a significant main effect of memory [SMT5 $>$ SMT1-4; $F_{(1,165)}=31.52, p<0.001$; and $F_{(1,165)}=9.79, p<0.002$; right and left PRC/ERC (Fig. 4E,F, respectively)] and by a significant task by memory interaction [SMT5 > SMT1-4 for DMS trials; $F_{(1,165)}=7.46 ; p=0.007$; right $\mathrm{PRC} / \mathrm{ERC}$ (Fig. $4 E)$ ]. Furthermore, there is a trend toward a main effect of task $\left(F_{(1,165)}=\right.$ $3.81, p=0.053)$ in the right PRC/ERC peak, indicating that the subsequent memory effect was greater for DMS trials than for CON trials (data not shown). As demonstrated in Figure 4, E and $F$, encoding-related activity in the PRC/ERC is sustained from the sample presentation period over the memory delay period and into the test presentation period. Activity in the right PHG/ mid-FG is also sustained if only nonmatch trials (single stimulus exposures) are considered for analysis (Fig. 4C). Overall, in DMS trials, the right $\mathrm{PHG} / \mathrm{mid}-\mathrm{FG}$ and the right and left PRC/ERC showed greater activation during the delay for samples that were subsequently remembered with high confidence than for those that were not.

An additional subsequent memory analysis allowed the same assessment separately for match and nonmatch trials, to control for the number of stimulus exposures. In nonmatch trials, sample stimuli were presented only once, but, in match trials, sample 
stimuli were presented twice. Because we were interested in long-term encodingrelated activation during the DMS delays that with high probability resulted in subsequent recollection, this is the most crucial analysis of our study.

This additional subsequent memory analysis on nonmatch trials confirmed that encoding-related activity in the right and left PRC/ERC [right peak at $x=34$, $y=-10, z=-28, Z=3.45, p<0.05_{\mathrm{COR}}$ (Fig. 5A, white arrows, $B$ ); left peak at $x=$ $-34, y=-12, z=-26, Z=2.05, p<$ $0.05_{\mathrm{COR}}$ (Fig. $5 A$, black arrow, $C$ ) ] and in the right $\mathrm{PHG} / \mathrm{mid}-\mathrm{FG}$ (Fig. $4 C$ ) is persistent across sample, memory delay, and test periods. Repeated-measures ANOVAs on DMS trials of VOI time series extracted from the right and left PRC/ERC were performed with the within-subject factors DMS trial (match vs nonmatch), memory (SMT5 vs SMT1-4), and event (sample vs delay vs test periods). This analysis revealed a significant main effect of memory [SMT5 > SMT1-4; $F_{(1,165)}=5.35, p=$ 0.022 (Fig. $5 B$ ); and $F_{(1,165)}=10.25, p=$ 0.002 (Fig. 5C); right and left PRC/ERC, respectively], a significant DMS trial by memory interaction [SMT5 $>$ SMT1-4 for nonmatch trials; $F_{(1,165)}=11.58, p=$ 0.001 (Fig. 5B); and $F_{(1,165)}=14.79, p<$ 0.001 (Fig. 5C); right and left PRC/ERC, respectively], and a trend toward a main effect of DMS trial [nonmatch $>$ match trials; $F_{(1,165)}=3.36 ; p=0.068$; right PRC/ERC (data not shown)]. The same analysis on the right PHG/mid-FG ROI also resulted in a significant trial by memory interaction [SMT5 $>$ SMT1-4 for nonmatch trials; $F_{(1,165)}=20.33 ; p<$ 0.001 (Fig. 4C)].

The separate subsequent memory analysis on nonmatch trials revealed an additional subsequent memory effect in the right hippocampal body [peak at $x=$ $34, y=-26, z=-14, Z=2.82, p<$ $0.05_{\mathrm{COR}}$ (Fig. $5 D$, white arrows) $]$ and the left hippocampal body [peak at $x=-30$, $y=-30, z=-12, Z=2.45, p<0.05_{\mathrm{COR}}$ (Fig. 5D, black arrow)]. VOI time-series repeated-measures ANOVAs in these two regions revealed a significant DMS trial by memory interaction in both foci [SMT5 $>$ SMT1-4 for nonmatch trials; right, $F_{(1,165)}=6.16, p=0.014$ (Fig. $5 E$ ); left, $F_{(1,165)}=7.64, p=0.006$ (Fig. $\left.5 F\right)$ ). There was also a main effect of memory $\left(\right.$ SMT5 $>$ SMT1 $\left.-4 ; F_{(1,165)}=13.97 ; p<0.001\right)$ in the left hippocampal body, indicating that activity is sustained throughout the DMS trial.

In summary, for nonmatch trials, in which a stimulus appears only once, a persistent subsequent memory effect as measured by DMS delay activity that is associated with subsequent recognition on the post-scan SMT was observed in the anterior (PRC/ERC) and posterior (PHG/mid-FG) parahippocampal gyrus, as well as
A RIGHT PRC/ERC

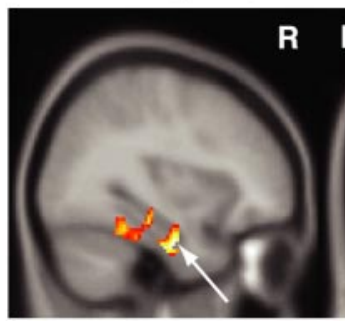

B RIGHT PRC/ERC

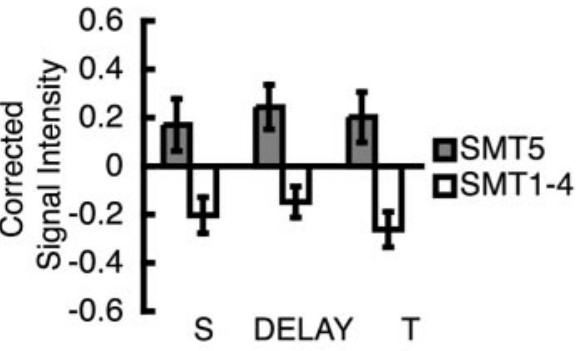

C LEFT PRC/ERC

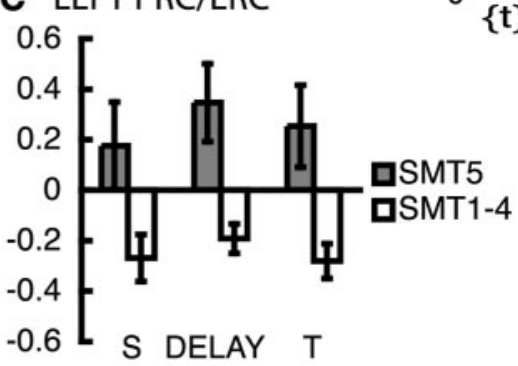

D RIGHT HIPP.BODY

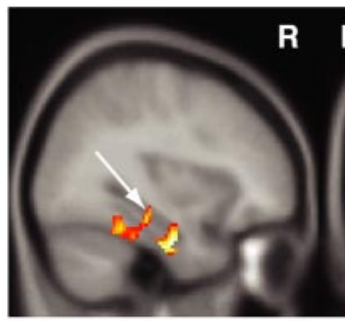

E RIGHT HIPP.BODY
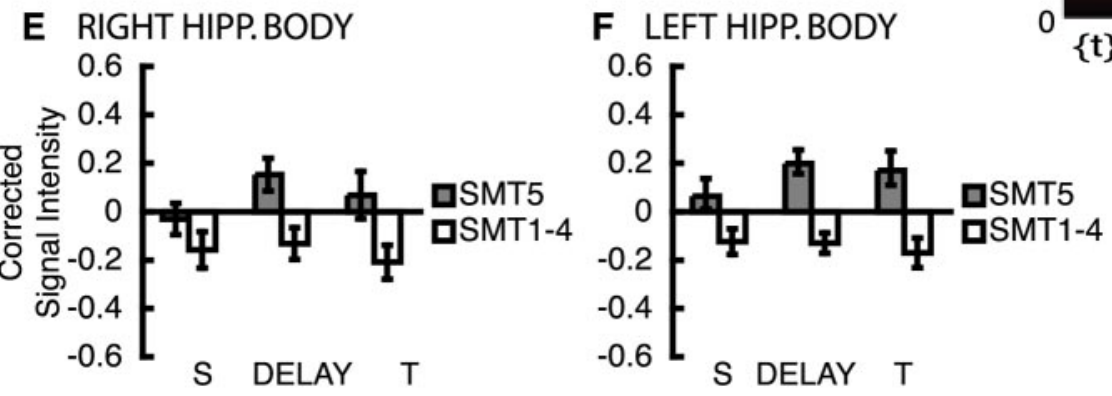

Figure 5. $\quad \mathrm{fMRI}$ results from subsequent memory analysis of nonmatch trials (single stimulus presentations). Please note that only activation within ROIs is superimposed on canonical average T1-weighted ICBM/MNI brain. A, Right PRC/ERC, $x=34, y=$ $-10, z=-28$ (white arrows); left PRC/ERC, $x=-34, y=-12, z=-26$ (black arrow). $B$, Corrected signal intensities during sample presentation (S), delay period (DELAY), and test presentation (T) from right PRC/ERC for DMS trials (nonmatch trials, single stimulus presentations); SMT5 > SMT1-4.C, Corrected signal intensities during sample, delay, and test presentation periods from left PRC/ERC for DMS nonmatch trials. D, Right hippocampal (HIPP.) body, $x=34, y=-26, z=-14$ (white arrows); left hippocampal body, $x=-30, y=30, z=-12$ (black arrow). E, Corrected signal intensities from right hippocampal body for DMS nonmatch trials (single stimulus presentations); SMT5 > SMT1-4.F, Corrected signal intensities from left hippocampal body for DMS nonmatch trials. $y$-Axes, Signal intensity grand mean scaled to 100 and global calculation using mean voxel value (within per image full mean/eight mask). R, Right; L, left. in the hippocampus, indicating that these regions play a role in successful encoding during the delay period of the DMS task. This persistent subsequent memory effect appears for nonmatch trials, in which sample stimuli were presented once before SMT performance, but not for match trials, when sample stimuli were presented twice before SMT performance.

Additional foci of activation, which showed activity during the delay periods of the DMS task that were associated with subsequent memory on the post-scan SMT task collapsed across all DMS trials, included the right inferior frontal gyrus (BA47), the left inferior frontal sulcus (BA45), the left posterior orbital gyrus 
(BA47), the left rolandic operculum (BA44), the gyrus rectus, the left and right insulae (BA13), the right and left superior parietal gyri (BA7), the right inferior occipital gyrus (BA19), the cuneus (BA18), and the caudate nucleus ( $p<0.01$, uncorrected; threshold extent, 5 voxels).

\section{Discussion}

This study found that parahippocampal and mid-FG regions were active during maintenance of trial-unique visual information during the delay component of a DMS task and, most importantly, that this delay period activity was correlated with postscan subsequent memory.

A bilateral region within the mid-FG/PHG was recruited for active maintenance of trial-unique visual information regardless of subsequent memory for sample stimuli. An analysis of signal intensities extracted from these regions and a subsequent memory multiple-regression analysis additionally revealed a subsequent memory effect in the right PHG/mid-FG. Our data provide a direct link between encoding and memory delay activity during delayed matching. Activity in the right PHG/mid-FG during the memory delay was greater for sample pictures that were subsequently remembered with high confidence than for those that were not. Furthermore, right anterior parahippocampal (perirhinal and possibly entorhinal cortex) and hippocampal regions showed a persistent subsequent memory effect across sample, memory delay, and test presentations periods of nonmatch trials (single stimulus presentations before subsequent memory task performance). The current fMRI findings in humans support the hypothesis that persistent activity within parahippocampal areas in the absence of stimulus input may enhance encoding. We derived this hypothesis from computational models of medial temporal long-term memory encoding (Jensen and Lisman, 1996; Hasselmo et al., 2002a,b; Koene et al., 2003).

In addition, these results extend previous neuroimaging studies of long-term encoding and subsequent memory (Brewer et al., 1998; Fernàndez et al., 1998, 1999; Wagner et al., 1998; Kirchhoff et al., 2000; Otten et al., 2001; Reber et al., 2002; Fletcher et al., 2003) by demonstrating that the activity associated with longterm subsequent memory persists in the absence of stimulus presentation. These results also extend previous neuroimaging studies of delayed matching (Ranganath et al., 2001; Stern et al., 2001) by demonstrating that delay-related activation observed during the DMS task in the MTL is directly linked to long-term encoding of stimuli that have no previous representation.

\section{Relationship to single-unit and intracellular recording studies and modeling of sustained activity}

The finding of sustained activity during delays in parahippocampal areas in humans is consistent with evidence for sustained unit activity in parahippocampal and entorhinal cortex during delayed matching tasks in nonhuman primates and in rats (Suzuki et al., 1997; Young et al., 1997). Recent intracellular recording data suggests a potential mechanism for mediating this sustained unit activity for novel stimuli in parahippocampal structures. Recordings in brain slice preparations of the entorhinal cortex demonstrate intrinsic cellular mechanisms activated by muscarinic cholinergic receptors that allow single neurons to maintain sustained spiking activity without synaptic transmission (Klink and Alsonso, 1997; Egorov et al., 2002). Detailed biophysical modeling of entorhinal neurons (Fransén et al., 2002) demonstrates that these mechanisms could underlie the delay period activity of single units recorded during performance of DMS and DNMS tasks (Suzuki et al., 1997; Young et al., 1997). These in- trinsic mechanisms could provide sustained activity to actively maintain representations of novel images for which no previous structured excitatory connectivity has been created, consistent with evidence for selective activation of medial temporal regions during delayed matching for novel stimuli (Ranganath et al., 2001; Stern et al., 2001).

As shown here, the activity during the delay period correlates with the subsequent memory for the stimuli. This is consistent with human memory studies suggesting the necessity of a buffer for holding information during encoding (Atkinson and Shiffrin, 1968; O’Reilly et al., 1999; Baddeley, 2000; Baddeley and Wilson, 2002). The active maintenance of novel stimuli in parahippocampal structures is essential in models of medial temporal circuits (Jensen and Lisman, 1996; Hasselmo et al., 2002a,b; Koene et al., 2003), to allow the encoding of slow behavioral events in the hippocampus using the fast time course of spike timingdependent synaptic plasticity (Levy and Steward, 1983; Markram et al., 1997; Bi and Poo, 2001). Computational models have used the mechanisms described in slice preparations of the entorhinal cortex (Klink and Alonso, 1997; Fransén et al., 2002) to allow a behavioral stimulus to cause repeated spiking over an extended period, even after the stimulus is no longer present (Jensen and Lisman, 1996; Fransen et al., 2002; Hasselmo et al., 2002a,b; Koene et al., 2003). In the models, this persistent spiking allows repeated modification of synapses between neurons that responded to behavioral input, thereby enhancing encoding. This is consistent with our fMRI data showing enhanced encoding associated with persistent activity in the perirhinal/entorhinal cortex during the delay period (Fig. $5 A, B$ ). This mechanism may constitute the cellular basis of an "episodic buffer" for holding information during encoding. Thus, the relationship between active maintenance during the delay and subsequent memory demonstrated in this study supports previous models of the processes necessary for encoding of long term memories.

\section{Relationship to previous neuroimaging studies of long-term encoding}

The foci of activation from our findings overlap with those reported in previous long-term encoding studies that demonstrated a subsequent memory effect in the PHG and FG for trialunique complex visual scenes (Brewer et al., 1998; Kirchhoff et al., 2000). Additional studies also observed a subsequent memory effect in the FG using words (Wagner et al., 1998). Consistent with our data, recent long-term encoding studies have shown a subsequent memory effect for word encoding in anterior parahippocampal areas, including the entorhinal (Fernàndez et al., 1999; Davachi et al., 2003) and the perirhinal cortex (Davachi and Wagner, 2002). Many previous neuroimaging studies of longterm encoding and subsequent memory also found MTL activations within the posterior PHG and the hippocampus for novel complex scenes and other visual information (Stern et al., 1996; Gabrieli et al., 1997; Kelley et al., 1998; Kirchhoff et al., 2000) and for word encoding (Fernàndez et al., 1998; Wagner et al., 1998; Otten et al., 2001; Reber et al., 2002).

We also observed long-term subsequent memory effects in the posterior PHG and in the hippocampus in this study, in particular during DMS delays of nonmatch trials, during which stimuli were presented only once before SMT performance. Together, these results suggest that regions active during encoding of sample stimuli during the DMS task delay overlap closely with those active during long-term encoding. 


\section{Relationship to previous neuroimaging studies of MTL involvement in delayed matching and working memory}

A previous study from our laboratory established that the hippocampus is active in subjects performing a "2-back" delayed matching task when trial-unique complex visual scenes were used as stimuli but not when stimuli were highly familiar (Stern et al., 2001). Behavioral data from a subsequent memory task used in that study provided evidence that subjects incidentally encoded the novel stimuli into LTM while performing the task at a similar rate as in our study. Together, these findings suggest that novel stimuli encountered during delayed matching are encoded into LTM.

Ranganath and D'Esposito (2001) also examined MTL activation during components of a DMS task. They assessed which MTL structures were active during a brief memory delay and compared this pattern with that observed during a separate longterm encoding control task with novel faces as stimuli. They reported a functional dissociation within the medial temporal lobes for delayed matching and long-term encoding tasks and suggested that the hippocampal activation observed during the delay period of their DMS task was attributable to active maintenance, not incidental encoding, and that the posterior PHG/mid-FG activation observed during the long-term encoding task was attributable to incidental encoding and not to active maintenance. The results of our study support the idea that the parahippocampal gyrus and the hippocampus are important for actively maintaining information across a delay (Ranganath and D'Esposito, 2001; Stern et al., 2001). However, rather than supporting a functional dissociation, our data provide evidence that parahippocampal structures and the hippocampus interact to enhance long-term encoding during delayed matching as shown by our subsequent memory data. Our data suggest a role of the hippocampus in active maintenance that also supports long-term encoding.

Our main finding that parahippocampal, hippocampal, and mid-fusiform regions can support successful encoding during delayed matching and during long-term encoding is perhaps similar to the idea that prefrontal regions can support working memory, long-term encoding (Gabrieli et al., 1998; Davachi et al., 2001; Ranganath et al., 2003; Rypma and D'Esposito, 2003), and episodic retrieval (Cabeza et al., 2002). Data from our laboratory have shown the involvement of both prefrontal and medial temporal areas in long-term encoding (Kirchhoff et al., 2000) and in short-term stimulus matching (Stern et al., 2001). However, data from our laboratory suggest that medial temporal areas may be more important for maintaining trial-unique stimuli during delayed matching, in contrast to working memory for familiar stimuli, which may primarily involve prefrontal cortex (Stern et al., 2001).

\section{Conclusions}

Our data demonstrate that the parahippocampal and midfusiform regions are important for actively maintaining novel information during brief memory delays and that this activation is directly linked to long-term encoding, as demonstrated by the subsequent memory effect observed during delayed matching. This indicates that delay activity in these regions can predict subsequent long-term memory on the SMT. These finding support the hypothesis derived from computational models of parahippocampal structures that active maintenance of novel stimuli enhances both delayed matching function and encoding of stimuli into long-term memory.

\section{References}

Atkinson RC, Shiffrin RM (1968) Human memory: a proposed system and its control processes. In: The psychology of learning and motivation (Spence KW, Spence JT, eds), pp 89-105. New York: Academic.

Baddeley A (2000) The episodic buffer: a new component of working memory? Trends Cogn Sci 4:417-423.

Baddeley A, Wilson BA (2002) Prose recall and amnesia: implications for the structure of working memory. Neuropsychologia 40:1737-1743.

Bi G, Poo M (2001) Synaptic modification by correlated activity: Hebb's postulate revisited. Annu Rev Neurosci 24:139-166.

Bi GQ, Poo MM (1998) Synaptic modifications in cultured hippocampal neurons: dependence on spike timing, synaptic strength, and postsynaptic cell type. J Neurosci 18:10464-10472.

Bortz J (1993) Statistik Für Sozialwissenschaftler, Ed 4, p 488. Berlin: Springer.

Boynton GM, Engel SA, Glover GH, Heeger DJ (1996) Linear systems analysis of functional magnetic resonance imaging in human V1. J Neurosci 16:4207-4221.

Brewer JB, Zhao Z, Desmond JE, Glover GH, Gabrieli JD (1998) Making memories: brain activity that predicts how well visual experience will be remembered. Science 281:1185-1187.

Buckner RL (1998) Event-related fMRI and the hemodynamic response. Hum Brain Mapp 6:373-377.

Cabeza R, Dolcos F, Graham R, Nyberg L (2002) Similarities and differences in the neural correlates of episodic memory retrieval and working memory. NeuroImage 16:317-330.

Cohen J, Cohen P (1983) Applied multiple regression/correlation for the behavioral sciences, Ed 2, pp 204-209. Hillsdale, NJ: Erlbaum.

Cohen JD, MacWhinney B, Flatt M, Provost J (1993) PsyScope: a new graphic interactive environment for designing psychology experiments. Behav Res Methods Instr Comp 25:257-271.

Courtney SM, Ungerleider LG, Keil K, Haxby JV (1997) Transient and sustained activity in a distributed neural system for human working memory. Nature 386:608-611.

Courtney SM, Petit L, Maisog JM, Ungerleider LG, Haxby JV (1998) An area specialized for spatial working memory in human frontal cortex. Science 279:1347-1351.

Dale AM, Buckner RL (1997) Selective averaging of rapidly presented individual trials using fMRI. Hum Brain Mapp 5:329-340.

Davachi L, Wagner AD (2002) Hippocampal contributions to episodic encoding: insights from relational and item-based learning. J Neurophysiol 88:982-990.

Davachi L, Maril A, Wagner AD (2001) When keeping in mind supports later bringing to mind: neural markers of phonological rehearsal predict subsequent remembering. J Cogn Neurosci 13:1059-1070.

Davachi L, Mitchell JP, Wagner AD (2003) Multiple routes to memory: distinct medial temporal lobe processes build item and source memories. Proc Natl Acad Sci USA 100:2157-2162.

Egorov AV, Hamam BN, Fransén E, Hasselmo ME, Alonso AA (2002) Graded persistent activity in entorhinal cortex neurons. Nature 420:173-178.

Elliott R, Dolan RJ (1999) Differential neural responses during performance of matching and nonmatching to sample tasks at two delay intervals. J Neurosci 19:5066-5073.

Fernàndez $\mathrm{G}$, Weyerts $\mathrm{H}$, Schrader-Bolsche $\mathrm{M}$, Tendolkar I, Smid HG, Tempelmann C, Hinrichs H, Scheich H, Elger CE, Mangun GR, Heinze HJ (1998) Successful verbal encoding into episodic memory engages the posterior hippocampus: a parametrically analyzed functional magnetic resonance imaging study. J Neurosci 18:1841-1847.

Fernàndez G, Brewer JB, Zhao Z, Glover GH, Gabrieli JD (1999) Level of sustained entorhinal activity at study correlates with subsequent cuedrecall performance: a functional magnetic resonance imaging study with high acquisition rate. Hippocampus 9:35-44.

Fletcher PC, Stephenson CM, Carpenter TA, Donovan T, Bullmorel ET (2003) Regional brain activations predicting subsequent memory success: an event-related fMRI study of the influence of encoding tasks. Cortex 39:1009-1026.

Fransén E, Alonso AA, Hasselmo ME (2002) Simulations of the role of the muscarinic-activated calcium-sensitive nonspecific cation current INCM in entorhinal neuronal activity during delayed matching tasks. J Neurosci 22:1081-1097.

Gabrieli JD, Brewer JB, Desmond JE, Glover GH (1997) Separate neural 
bases of two fundamental memory processes in the human medial temporal lobe. Science 276:264-266.

Gabrieli JD, Poldrack RA, Desmond JE (1998) The role of left prefrontal cortex in language and memory. Proc Natl Acad Sci USA 95:906-913.

Geisser S, Greenhouse SW (1958) An extension of Box's results on the use of the F-distribution in multivariate analysis. Ann Math Stat 29:885-891.

Hasselmo ME, Hay J, Ilyn M, Gorchetchnikov A (2002a) Neuromodulation, theta rhythm and rat spatial navigation. Neural Netw 15:689-707.

Hasselmo ME, Cannon RC, Koene RA (2002b) A simulation of parahippocampal and hippocampal structures guiding spatial navigation of a virtual rat in a virtual environment: a functional framework for theta theory. In: The parahippocampal region: organization and role in cognitive functions (Witter MP, Wouterlood FG, eds), pp 139-161. Oxford: Oxford UP.

Howard MW, Kahana MJ (2002) A distributed representation of temporal context. J Math Psychol 46:269-299.

Insausti R, Juottonen K, Soininen H, Insausti AM, Partanen K, Vainio P, Laakso MP, Pitkanen A (1998) MR volumetric analysis of the human entorhinal, perirhinal, and temporopolar cortices. AJNR Am J Neuroradiol 19:659-671.

Jensen O, Lisman JE (1996) Novel lists of $7+/-2$ known items can be reliably stored in an oscillatory short-term memory network: interaction with long-term memory. Learn Mem 3:257-263.

Kelley WM, Miezin FM, McDermott KB, Buckner RL, Raichle ME, Cohen NJ, Ollinger JM, Akbudak E, Conturo TE, Snyder AZ, Petersen SE (1998) Hemispheric specialization in human dorsal frontal cortex and medial temporal lobe for verbal and nonverbal memory encoding. Neuron 20:927-936.

Kirchhoff BA, Wagner AD, Maril A, Stern CE (2000) Prefrontal-temporal circuitry for episodic encoding and subsequent memory. J Neurosci 20:6173-6180.

Klink R, Alonso A (1997) Muscarinic modulation of the oscillatory and repetitive firing properties of entorhinal cortex layer II neurons. J Neurophysiol 77:1813-1828.

Koene RA, Gorchetchnikov A, Cannon RC, Hasselmo ME (2003) Modeling goal-directed spatial navigation in the rat based on physiological data from the hippocampal formation. Neural Netw 16:577-584.

Levy WB, Steward O (1983) Temporal contiguity requirements for longterm associative potentiation/depression in the hippocampus. Neuroscience 8:791-797.

Maldjian JA, Laurienti PJ, Kraft RA, Burdette JH (2003) An automated method for neuroanatomic and cytoarchitectonic atlas-based interrogation of fMRI data sets. NeuroImage 19:1233-1239.

Markram H, Lubke J, Frotscher M, Sakmann B (1997) Regulation of synaptic efficacy by coincidence of postsynaptic APs and EPSPs. Science 275:213-215.

Mazziotta J, Toga A, Evans A, Fox P, Lancaster J, Zilles K, Woods R, Paus T, Simpson G, Pike B, Holmes C, Collins L, Thompson P, MacDonald D, Iacoboni M, Schormann T, Amunts K, Palomero-Gallagher N, Geyer S, Parsons L, et al. (2001) A probabilistic atlas and reference system for the human brain: International Consortium for Brain Mapping (ICBM). Philos Trans R Soc Lond B Biol Sci 356:1293-1322.

O'Reilly RC, Braver TS, Cohen JD (1999) A biologically based computa- tional model of working memory. In: Models of working memory. Mechanisms of active maintenance and executive control (Miyake A, Shah P, eds), pp 375-411. New York: Cambridge UP.

Otten LJ, Henson RN, Rugg MD (2001) Depth of processing effects on neural correlates of memory encoding: relationship between findings from across- and within-task comparisons. Brain 124:399-412.

Postle BR, D’Esposito M (1999a) "What"-Then-Where“ in visual working memory: an event-related fMRI study. J Cognit Neurosci 11:585-597.

Postle BR, D’Esposito M (1999b) Dissociation of human caudate nucleus activity in spatial and nonspatial working memory: an event-related fMRI study. Brain Res Cogn Brain Res 8:107-115.

Postle BR, Zarahn E, D’Esposito M (2000) Using event-related fMRI to assess delay-period activity during performance of spatial and nonspatial working memory tasks. Brain Res Brain Res Protoc 5:57-66.

Pruessner JC, Kohler S, Crane J, Pruessner M, Lord C, Byrne A, Kabani N, Collins DL, Evans AC (2002) Volumetry of temporopolar, perirhinal, entorhinal and parahippocampal cortex from high-resolution MR images: considering the variability of the collateral sulcus. Cereb Cortex 12:1342-1353.

Ranganath C, D’Esposito M (2001) Medial temporal lobe activity associated with active maintenance of novel information. Neuron 31:865-873.

Ranganath C, Johnson MK, D'Esposito M (2003) Prefrontal activity associated with working memory and episodic long-term memory. Neuropsychologia 41:378-389.

Reber PJ, Siwiec RM, Gitelman DR, Parrish TB, Mesulam MM, Paller KA, Gitleman DR (2002) Neural correlates of successful encoding identified using functional magnetic resonance imaging. J Neurosci 22:9541-9548.

Rypma B, D'Esposito M (2003) A subsequent-memory effect in dorsolateral prefrontal cortex. Brain Res Cogn Brain Res 16:162-166.

Sherman SJ, Atri A, Hasselmo ME, Stern CE, Howard MW (2003) Scopolamine impairs human recognition memory: data and modeling. Behav Neurosci 117:526-539.

Stern CE, Corkin S, Gonzalez RG, Guimaraes AR, Baker JR, Jennings PJ, Carr CA, Sugiura RM, Vedantham V, Rosen BR (1996) The hippocampal formation participates in novel picture encoding: evidence from functional magnetic resonance imaging. Proc Natl Acad Sci USA 93:8660-8665

Stern CE, Sherman SJ, Kirchhoff BA, Hasselmo ME (2001) Medial temporal and prefrontal contributions to working memory tasks with novel and familiar stimuli. Hippocampus 11:337-346.

Suzuki WA, Miller EK, Desimone R (1997) Object and place memory in the macaque entorhinal cortex. J Neurophysiol 78:1062-1081.

Wagner AD, Schacter DL, Rotte M, Koutstaal W, Maril A, Dale AM, Rosen BR, Buckner RL (1998) Building memories: remembering and forgetting of verbal experiences as predicted by brain activity. Science 281:1188-1191.

Yonelinas AP (1994) Receiver-operating characteristics in recognition memory: evidence for a dual-process model. J Exp Psychol Learn Mem Cogn 20:1341-1354.

Young BJ, Otto T, Fox GD, Eichenbaum H (1997) Memory representation within the parahippocampal region. J Neurosci 17:5183-5195.

Zarahn E, Aguirre G, D’Esposito M (1997) A trial-based experimental design for fMRI. NeuroImage 6:122-138. 